PROCEEDINGS OF THE AMERICAN MATHEMATICAL SOCIETY

Volume 124, Number 2, February 1996

\title{
INTEGRAL CONDITIONS ON THE ASYMPTOTIC STABILITY FOR THE DAMPED LINEAR OSCILLATOR WITH SMALL DAMPING
}

\author{
L. HATVANI
}

(Communicated by Hal L. Smith)

\begin{abstract}
The equation $x^{\prime \prime}+h(t) x^{\prime}+k^{2} x=0$ is considered under the assumption $0 \leq h(t) \leq \bar{h}<\infty(t \geq 0)$. It is proved that $\lim \sup _{t \rightarrow \infty}\left(t^{-2 / 3} \int_{0}^{t} h\right)>0$ is sufficient for the asymptotic stability of $x=x^{\prime}=0$, and $2 / 3$ is best possible here. This will be a consequence of a general result on the intermittent damping, which means that $h$ is controlled only on a sequence of non-overlapping intervals.
\end{abstract}

\section{INTRODUCTION AND REMARKS}

The motion of the damped linear oscillator of one degree of freedom is described by the equation

$$
x^{\prime \prime}+h(t) x^{\prime}+k^{2} x=0,
$$

where $h: \mathbf{R}_{+}:=[0, \infty) \rightarrow \mathbf{R}_{+}$is a piecewise continuous function (the damping coefficient) and $k$ is a positive constant. It is an old problem in the theory of ordinary differential equations to find sufficient conditions for the asymptotic stability of the equilibrium state $x=x^{\prime}=0$; i.e., conditions guaranteeing $\lim _{t \rightarrow \infty} x(t)=\lim _{t \rightarrow \infty} x^{\prime}(t)=0$ for every solution $x$ of equation (1.1).

It is well known from the elements that this is the case if $0<h(t) \equiv h_{0}=$ const. J. J. Levin and J. A. Nohel [10] proved that $0<\underline{h} \leq h(t) \leq \bar{h}<\infty\left(t \in \mathbf{R}_{+} ; \underline{h}, \bar{h}\right.$ are constant) is also sufficient for this property. After this result the investigators tried to weaken at least one of these two boundedness conditions [1-3,5-9,11-15]. Accordingly, two cases have been distinguished: the case of "large damping" $0<$ $\underline{h} \leq h(t)<\infty\left(t \in \mathbf{R}_{+}\right)$and the case of "small damping" $0 \leq h(t) \leq \bar{h}<\infty$ $\left(t \in \mathbf{R}_{+}\right)$. They require different methods. For large damping one has to exclude the phenomenon of overdamping, i.e., the existence of a solution $x$ with $\lim _{t \rightarrow \infty} x(t)=$ $x_{*} \neq 0$. In the case of small damping the lack of the asymptotic stability can be caused by an oscillation not tending to the equilibrium state $x=x^{\prime}=0$.

Received by the editors January 7, 1994

1991 Mathematics Subject Classification. Primary 34D20, 34A30.

Key words and phrases. Intermittent damping, energy method.

The author was supported by the Hungarian National Foundation for Scientific Research with grant number 1157 .

(C)1996 American Mathematical Society 
Considering the case of large damping Z. Artstein and E. F. Infante [1] proved that the inequality

$$
\int_{0}^{t} h(s) d s \leq A+B t^{2} \quad\left(t \in \mathbf{R}_{+} ; A, B=\text { const. }\right)
$$

implies asymptotic stability, and the exponent 2 is best possible. R. A. Smith [14] gave a necessary and sufficient condition similarly for the case of large damping in terms of the integral of $h$, but it is not easy to check it. (For an easy-to-check necessary and sufficient integral condition see [5].)

The case of small damping is more delicate. R. Ballieu and K. Peiffer [2] proved that $\int_{0}^{\infty} h=\infty$ is necessary and sufficient for the asymptotic stability, provided that $h$ is monotonous. However, there is no necessary and sufficient integral condition for the general (non-monotonous) case.

Recently P. Pucci and J. Serrin published a series of papers [11-13] on the asymptotic stability of the equilibria of damped mechanical systems. They investigated very general nonlinear systems with many degrees of freedom. Most of their theorems work both for the small and large damping. The restriction of a typical result of theirs to equation (1.1) says: if $h$ satisfies an inequality $0 \leq \sigma(t) \leq h(t) \leq \delta(t)$ $\left(t \in \mathbf{R}_{+}\right)$with some functions $\sigma, \delta$ such that $\sigma \delta$ is bounded, and either $\sigma$ is of bounded variation or $\log \sigma$ is uniformly Lipschitz on $\mathbf{R}_{+}$, then $\int_{0}^{\infty} \sigma=\infty$ is sufficient for the asymptotic stability of the equilibrium $x=x^{\prime}=0$ of (1.1) (see [11, Theorem A]).

The aim of this paper is to give integral conditions (without any additional monotony-like restriction) on $h$ guaranteeing asymptotic stability for the equilibrium $x=x^{\prime}=0$ of (1.1) in the case of small damping.

It is well known that $\int_{0}^{\infty} h=\infty$ alone is not sufficient, in general, for the asymptotic stability (see $[6,8,9,13]$ and Example 2.3 of the present paper). A. G. Surkov [15] proved that in the case of small damping

$$
\int_{0}^{t} h(s) d s \geq B t \quad \text { for } t \text { large enough; } 0<B=\text { const. }
$$

already implies asymptotic stability. The following question arises: Is the exponent 1 on the right-hand side best possible? We will show that the answer is "no". The exponent 1 can be changed for $2 / 3$, but $2 / 3$ is best possible. To be more precise, the following theorem, analogous with that of Artstein and Infante for the large damping, can be proved:

Theorem 1.1. Suppose that the condition

$$
0 \leq h(t) \leq \bar{h}<\infty
$$

is satisfied for all $t \in \mathbf{R}_{+}$with some constant $\bar{h}$.

(A) If

$$
\limsup _{t \rightarrow \infty}\left(t^{-2 / 3} \int_{0}^{t} h(s) d s\right)>0,
$$

then the zero solution of (1.1) is asymptotically stable.

(B) For every $\varepsilon>0$ there exists a function $h$ such that

$$
\liminf _{t \rightarrow \infty}\left(t^{-(2 / 3-\varepsilon)} \int_{0}^{t} h(s) d s\right)>0 ;
$$


nevertheless, the zero solution of (1.1) is not asymptotically stable.

This theorem will be a consequence of a result about intermittent damping. By this type of damping we mean that certain restrictions or controls are placed on $h$ on a sequence of non-overlapping intervals $I_{n}=\left(a_{n}, b_{n}\right)$ of $\mathbf{R}_{+}$, with $a_{n} \rightarrow \infty$; on the other hand, in the gaps between these intervals the damping coefficient $h$ is assumed to be uncontrolled $[6,8,13,14]$. (Of course, in the case of small damping the damping coefficient $h$ is assumed to be bounded on the whole $\mathbf{R}_{+}$.)

It is known [14] that if $0<\underline{h} \leq h(t) \leq \bar{h}<\infty$ on $I=\bigcup_{n=1}^{\infty} I_{n}$, then

$$
\sum_{n=1}^{\infty}\left|I_{n}\right|^{3}=\infty
$$

implies asymptotic stability, where $\left|I_{n}\right|$ denotes the length of $I_{n}$. Moreover, the exponent 3 is best possible [13]. We generalize this result to the general case of small damping.

Theorem 1.2. Suppose that (1.3) is satisfied. If there exists a sequence $\left\{I_{n}\right\}$ of non-overlapping intervals such that

$$
\sum_{n=1}^{\infty} \frac{1}{1+\left|I_{n}\right|^{2}}\left(\int_{I_{n}} h(s) d s\right)^{3}=\infty,
$$

then the zero solution of (1.1) is asymptotically stable.

Corollary 1.3. Suppose that (1.3) is satisfied. If there exists a sequence $\left\{I_{n}\right\}$ of non-overlapping intervals such that $\left\{\left|I_{n}\right|\right\}$ is bounded and

$$
\sum_{n=1}^{\infty}\left(\int_{I_{n}} h(s) d s\right)^{3}=\infty
$$

then the zero solution of (1.1) is asymptotically stable. Moreover, the exponent 3 in the statement is best possible.

Corollary 1.4. Suppose that (1.3) is satisfied. If there exist a sequence $\left\{I_{n}\right\}$ of non-overlapping intervals and a constant $\delta>0$ such that

$$
\frac{1}{1+\left|I_{n}\right|^{2 / 3}} \int_{I_{n}} h(s) d s \geq \delta, \quad n=1,2, \ldots,
$$

then the zero solution of (1.1) is asymptotically stable. Moreover, the exponent 2/3 in the statement is best possible.

It can be noticed that the earlier results $[8,13,14]$ used the condition $\inf _{I_{n}} h>$ $0(n=1,2, \ldots)$ which is not supposed here.

\section{The PROOFS}

We will use the basic theorem of [6], which needs the following concept:

Definition 2.1. Let a constant $\alpha>0$ be given. A sequence $A=\left\{\tau_{n}\right\}, 0 \leq \tau_{n}<$ $\tau_{n+1}, n=1,2, \ldots$, is said to be asymptotically $\alpha$-discrete if

$$
\liminf _{n \rightarrow \infty}\left(\tau_{n+1}-\tau_{n}\right) \geq \alpha
$$


Theorem 2.2 ( [6, Theorem 4.1]). Suppose that (1.3) is satisfied. If

$$
\int_{0}^{\infty} h(t)[\operatorname{dist}(t, A)]^{2} d t=\infty
$$

for every asymptotically $(\pi / k)$-discrete set $A$, then the zero solution of (1.1) is asymptotically stable.

Proof of Theorem 1.2. Suppose that we are given a sequence $\left\{I_{n}\right\}$ with property (1.6) and an arbitrary $(\pi / k)$-discrete set $A=\left\{\tau_{n}\right\}$. We show that (2.1) holds; i.e.

$$
\sum_{n=1}^{\infty} \int_{\tau_{n}}^{\tau_{n+1}} h(t)\left[\min \left\{t-\tau_{n}, \tau_{n+1}-t\right\}\right]^{2} d t=\infty .
$$

Without loss of the generality we may assume that

$$
\pi / 2 k \leq \tau_{n+1}-\tau_{n} \leq 2 \pi / k \text { for } n=1,2, \ldots
$$

In fact, this inequality is satisfied for all $n>N$ with a suitable $N$, and we may drop the members $\tau_{1}, \ldots, \tau_{N}$. On the other hand, if we add new members (greater than $\tau_{1}$ ) to set $A$, then the integral in (2.1) decreases. This means that it is enough to show (2.1) for all the asymptotically $(\pi / k)$-discrete sets $A$ satisfying (2.3).

We divide the proof into four steps.

Step 1. We estimate the $n$-th member of the infinite sum in (2.2) by the integral $H_{n}:=\int_{\tau_{n}}^{\tau_{n+1}} h$. To this end, define $\alpha_{n}:=H_{n} / 8 \bar{h}$. By (1.3) and (2.3) we have $\alpha_{n} \leq \pi / 4 k$ and

$$
\begin{aligned}
& \int_{\tau_{n}}^{\tau_{n+1}} h(t)\left[\min \left\{t-\tau_{n} ; \tau_{n+1}-t\right\}\right]^{2} d t \geq \alpha_{n}^{2} \int_{\tau_{n}+\alpha_{n}}^{\tau_{n+1}-\alpha_{n}} h(t) d t \\
& \quad \geq \alpha_{n}^{2}\left(H_{n}-2 \alpha_{n} \bar{h}\right) \geq \alpha_{n}^{2} H_{n} / 2=H_{n}^{3} / 2^{7}(\bar{h})^{2}=c_{1}\left(\int_{\tau_{n}}^{\tau_{n+1}} h(t) d t\right)^{3}
\end{aligned}
$$

(here, and in the sequel, $c_{i}, i=1,2, \ldots$, denote appropriate constants).

Step 2. In order to make it easier to estimate the infinite sum in (2.2) by the infinite sum in (1.6), we modify sequence $\left\{I_{n}\right\}$.

Suppose that interval $I_{j}=\left(a_{j}, b_{j}\right)$ contains at least one element of set $A$. Let $m_{j}$ and $n_{j}$ denote the smallest and the largest integer, respectively, such that $m_{j} \leq n_{j}$ and $\left[\tau_{m_{j}}, \tau_{n_{j}}\right] \subset I_{j}$. Change interval $I_{j}$ to that of

$$
I_{j}^{1}:=\left(a_{j}, \tau_{m_{j}}\right), I_{j}^{2}:=\left(\tau_{m_{j}}, \tau_{n_{j}}\right), I_{j}^{3}=\left(\tau_{n_{j}}, b_{j}\right)
$$

over which the integral of $h$ is maximal. (The new interval will be denoted by $I_{j}$ again.) The intervals in $\left\{I_{n}\right\}$ containing no elements of $A$ remain unchanged. It is easy to see that after the changes condition (1.6) remains satisfied. On the other hand, taking an interval $\left[\tau_{n}, \tau_{n+1}\right]$ whose intersection with $I:=\bigcup_{j=1}^{\infty} I_{j}$ is not empty, we have two possibilities:

(a) The open interval $\left(\tau_{n}, \tau_{n+1}\right)$ is not covered by the union $I$.

(b) The open interval $\left(\tau_{n}, \tau_{n+1}\right)$ is covered by an interval $I_{m}$ of the sequence $\left\{I_{j}\right\}$, and there are integers $n_{m} \leq n, r_{m} \geq 1$ such that $I_{m}=\left(\tau_{n_{m}}, \tau_{n_{m}+r_{m}}\right)$. 
Step 3. We estimate $\int_{\tau_{n}}^{\tau_{n+1}} h$ in case (a). If $I_{j_{n}}, I_{j_{n}+1}, \ldots, I_{j_{n}+\ell_{n}}\left(\ell_{n} \geq 0\right)$ are all the intervals contained by $\left[\tau_{n}, \tau_{n+1}\right]$, then we have

$$
\left(\int_{\tau_{n}}^{\tau_{n+1}} h\right)^{3} \geq\left(\sum_{m=j_{n}}^{j_{n}+\ell_{n}} \int_{I_{m}} h\right)^{3} \geq \sum_{m=j_{n}}^{j_{n}+\ell_{n}} \frac{1}{1+\left|I_{m}\right|^{2}}\left(\int_{I_{m}} h\right)^{3} .
$$

Step 4. We estimate $\int_{\tau_{n m}}^{\tau_{n_{m}+r_{m}}} h$ in case (b). Since the function $y=x^{3}$ is convex, we have $[4$, Theorem 86$]$ the inequality

$$
\left(\frac{x_{1}+\ldots+x_{r}}{r}\right)^{3} \leq \frac{x_{1}^{3}+\ldots+x_{r}^{3}}{r} \quad\left(x_{i} \geq 0\right) .
$$

Therefore, using also (2.3) we obtain

$$
\begin{gathered}
\sum_{n=n_{m}}^{n_{m}+r_{m}-1}\left(\int_{\tau_{n}}^{\tau_{n+1}} h\right)^{3} \geq \frac{1}{r_{m}^{2}}\left(\sum_{n=n_{m}}^{n_{m}+r_{m}-1} \int_{\tau_{n}}^{\tau_{n+1}} h\right)^{3}=\frac{1}{r_{m}^{2}}\left(\int_{I_{m}} h\right)^{3} \\
\geq \frac{\pi^{2}}{4 k^{2}\left(\tau_{n_{m}+r_{m}}-\tau_{n_{m}}\right)^{2}}\left(\int_{I_{m}} h\right)^{3} \geq c_{2} \frac{1}{1+\left|I_{m}\right|^{2}}\left(\int_{I_{m}} h\right)^{3} .
\end{gathered}
$$

By (2.4) and the estimates of Steps 3 and 4, condition (1.6) implies (2.1). Theorem 1.2 is proved.

Proof of statement (A) in Theorem 1.1. By Theorem 1.2 it is enough to prove that from (1.4) there follows the existence of a sequence of non-overlapping intervals $I_{n}$, $n=1,2, \ldots$, and a $\gamma>0$ such that

$$
\frac{1}{1+\left|I_{n}\right|^{2}}\left(\int_{I_{n}} h\right)^{3} \geq \gamma, \quad n=1,2, \ldots
$$

If (1.4) holds, then there is a $\delta>0$ such that

$$
\frac{1}{t^{2 / 3}} \int_{0}^{t} h(s) d s \geq \delta
$$

for some $t$ arbitrarily large. Let $\gamma:=(\delta / 4)^{3}$. We will show that for an arbitrary $a \geq 0$ there exists a real number $b \geq a+1$ such that (2.5) is satisfied with $\gamma$ and $I_{n}=(a, b)$. In fact, choose $b$ so large that

$$
\frac{1}{b^{2 / 3}} \int_{0}^{a} h<\frac{\delta}{2}, \quad \frac{1}{b^{2 / 3}} \int_{0}^{b} h \geq \delta
$$

are satisfied. Then we have

$$
\left(\frac{1}{1+(b-a)^{2}}\right)^{1 / 3} \int_{a}^{b} h \geq \frac{1}{2} \frac{1}{b^{2 / 3}}\left(\int_{0}^{b} h-\int_{0}^{a} h\right) \geq \frac{\delta}{2}-\frac{\delta}{4}=\frac{\delta}{4}=\gamma^{1 / 3}
$$

i.e., (2.5) is satisfied for $I_{n}=(a, b)$.

Statement (A) in Theorem 1.1 is proved.

It remains to prove that the exponents in statement (A) of Theorem 1.1 and in Corollaries 1.3-1.4 are best possible. To this end we need a simplified version of some earlier constructions ([13, Proposition 1]; [6, Example 4.3]; [8, Theorem 2]). 
Example 2.3. Consider the differential equation

$$
x^{\prime \prime}+h_{*}(t) x^{\prime}+x=0
$$

with an on-off damping function $h_{*}$ defined by

$$
h_{*}(t)= \begin{cases}1, & \text { if } t_{n} \leq t \leq t_{n}+1 / n^{\alpha}, \quad n=1,2, \ldots, \\ 0, & \text { otherwise }\end{cases}
$$

It is supposed that $1 / 3<\alpha<1$, and $t_{n+1}-t_{n} \geq n^{-\alpha}$ for $n=1,2, \ldots$.

Rewrite equation (2.6) into the equivalent system

$$
x^{\prime}=y, \quad y^{\prime}=-x-h_{*}(t) y,
$$

which in polar coordinates $r, \varphi(x=r \cos \varphi, y=r \sin \varphi)$ reads as follows:

$$
\varphi^{\prime}=-1-h_{*}(t) \sin \varphi \cos \varphi, r^{\prime}=-h_{*}(t) r \sin ^{2} \varphi .
$$

Let $x_{*}$ denote the smooth solution of the initial value problem

$$
x^{\prime \prime}+h_{*}(t) x^{\prime}+x=0 ; \quad x\left(t_{1}\right)=1, x^{\prime}\left(t_{1}\right)=0 .
$$

We denote by $r_{*}, \varphi_{*}$ the corresponding polar functions.

Proposition 2.4. There is a sequence $\left\{t_{n}\right\}$ such that

$$
\pi \leq t_{n+1}-t_{n} \leq \pi+O\left(n^{-\alpha}\right), \quad n=1,2, \ldots,
$$

and the $t_{n}$ 's are exactly the values of variable $t$ where $\sin \varphi_{*}(t)=0$. Moreover, $r_{*}(t) \not 0$ as $t \rightarrow \infty$; i.e., the equilibrium $x=x^{\prime}=0$ of (2.6) is not asymptotically stable.

Proof. Let $\psi$ denote the solution of the initial value problem

$$
\varphi^{\prime}=-1-\sin \varphi \cos \varphi ; \quad \varphi(0)=0 .
$$

Obviously,

$$
-t \leq \psi(t) \leq-t / 2 \text { for } t \in[0, \pi / 2] .
$$

Now we can choose $\left\{t_{n}\right\}$ inductively as follows. Let $t_{1} \geq 0$ be arbitrary, and suppose that $t_{1}, t_{2}, \ldots, t_{n}$ are defined according to the requirements of Proposition 2.4. Then define $t_{n+1}$ by

$$
t_{n+1}:=t_{n}+\frac{1}{n^{\alpha}}+\left(\pi-\left|\psi\left(\frac{1}{n^{\alpha}}\right)\right|\right) .
$$

From (2.11) we obtain

$$
\frac{1}{2 n^{\alpha}} \leq\left|\psi\left(\frac{1}{n^{\alpha}}\right)\right| \leq \frac{1}{n^{\alpha}}
$$

whence we have

$$
\pi \leq t_{n+1}-t_{n} \leq \pi+\frac{1}{2 n^{\alpha}} .
$$

It is easy to see that $\varphi_{*}(t)=\varphi\left(t_{n}\right)+\psi\left(t-t_{n}\right)$ for $t \in\left[t_{n}, t_{n}+1 / n^{\alpha}\right]$; furthermore, $t_{n}$ and $t_{n+1}$ are consecutive zeros of $\sin \varphi_{*}$. 
On the other hand, by the second equation of (2.8) and estimate (2.12) we have

$$
\begin{aligned}
\ln \frac{r_{*}\left(t_{n+1}\right)}{r_{*}\left(t_{n}\right)} & =\int_{t_{n}}^{t_{n+1}} \frac{r_{*}^{\prime}(t)}{r_{*}(t)} d t=-\int_{t_{n}}^{t_{n}+1 / n^{\alpha}} \sin ^{2} \varphi_{*}(t) d t \\
& =-\int_{0}^{1 / n^{\alpha}} \sin ^{2} \psi(s) d s \geq-\frac{1}{n^{3 \alpha}} .
\end{aligned}
$$

Since $\alpha>1 / 3$ this means that $r_{*}(t) \not 0$ as $t \rightarrow \infty$.

Proposition 2.4 is proved.

Proof of statement (B) in Theorem 1.1 and of Corollaries 1.3-1.4. We have only to prove that the exponents are best possible. For an arbitrary $\varepsilon \in(0,2 / 3)$ fix $\alpha$ such that $1 / 3<\alpha<1 /(3-\varepsilon)$, and consider the damping coefficient (2.7) with $\left\{t_{n}\right\}$ guaranteed by Proposition 2.4. Then the solution of (2.9) does not tend to zero as $t \rightarrow \infty$; therefore, the equilibrium is not asymptotically stable. On the other hand, all the conditions weakened by modifying the exponents by $\varepsilon$ are satisfied.

In fact, this is obvious in the case of Corollary 1.3 by setting $I_{n}=\left(t_{n}, t_{n+1}\right)$ and taking into account $\sum_{n=1}^{\infty} 1 / n^{(3-\varepsilon) \alpha}=\infty$.

For the further cases we use the estimate

$$
\int_{t_{m}}^{t_{n}} h_{*}=\sum_{i=m}^{n-1} \frac{1}{i^{\alpha}} \geq \frac{n^{1-\alpha}-m^{1-\alpha}}{1-\alpha}, \quad n>m .
$$

From this, using also (2.10) we obtain

$$
\frac{1}{t_{n}^{2 / 3-\varepsilon}} \int_{0}^{t_{n}} h_{*} \geq c_{3} \frac{1}{\left(t_{n}-t_{1}\right)^{2 / 3-\varepsilon}} \int_{t_{1}}^{t_{n}} h_{*} \geq c_{4} \frac{n^{1-\alpha}-1}{n^{2 / 3-\varepsilon}} \geq c_{5}>0
$$

with appropriate constants $c_{3}, c_{4}, c_{5}$ for all $n=1,2, \ldots$ This concludes the proof of statement (B) in Theorem 1.1.

Similarly, for an arbitrarily fixed $\delta>0$ we have

$$
\frac{1}{1+\left(t_{n}-t_{m}\right)^{2 / 3-\varepsilon}} \int_{t_{m}}^{t_{n}} h_{*} \geq c_{6} \frac{n^{1-\alpha}-m^{1-\alpha}}{(n-m)^{2 / 3-\varepsilon}} \geq \delta
$$

for every natural $m$ with sufficiently large $n=n(m)$. This means that a sequence $\left\{I_{j}\right\}$ of non-overlapping intervals can be defined such that

$$
\frac{1}{1+\left|I_{j}\right|^{2 / 3-\varepsilon}} \int_{I_{j}} h_{*} \geq \delta, \quad j=1,2, \ldots,
$$

are satisfied, proving Corollary 1.4.

\section{ACKNOWLEDGMENT}

I am very grateful to my colleagues V. Totik and T. Krisztin for valuable discussions.

\section{REFERENCES}

1. Z. Artstein and E. F. Infante, On the asymptotic stability of oscillators with unbounded damping, Quart. Appl. Mech. 34 (1976), 195-199. MR 57:6665

2. R. J. Ballieu and K. Peiffer, Attractivity of the origin for the equation $\ddot{x}+f(t, x, \dot{x})|\dot{x}|^{\alpha} \dot{x}+$ $g(x)=0$, J. Math. Anal. Appl. 34 (1978), 321-332. MR 80a:34057

3. T. A. Burton and J. W. Hooker, On solutions of differential equations tending to zero, J. Reine Angew. Math. 267 (1974), 151-165. MR 50:702 
4. G. H. Hardy, J. E. Littlewood, and G. Pólya, Inequalities, Cambridge Univ. Press, Cambridge, 1952. MR 13:727e

5. L. Hatvani, T. Krisztin, and V. Totik, A necessary and sufficient condition for the asymptotic stability of the damped oscillator, J. Differential Equations (to appear).

6. L. Hatvani and V. Totik, Asymptotic stability of the equilibrium of the damped oscillator, Differential Integral Equations 6 (1993), 835-848. MR 94c:34080

7. J. Karsai, On the global asymptotic stability of the zero solution of the equation $\ddot{x}+g(t, x, \dot{x}) \dot{x}+$ $f(x)=0$, Studia Sci. Math. Hungar. 19 (1984), 385-393. MR 87m:34064

8. _ On the asymptotic stability of the zero solution of certain nonlinear second order differential equations, Differential Equations: Qualitative Theory (Szeged, 1984), Colloq. Math. Soc. János Bolyai, vol. 47, North-Holland, Amsterdam and New York, 1987, pp. 495-503. MR 88e: 34087

9. V. Kertész, Stability investigation of the differential equation of damped oscillation, Alkalmaz. Mat. Lapok 8 (1982), 323-339. (Hungarian, English summary) MR 85h:34058b

10. J. J. Levin and J. A. Nohel, Global asymptotic stability of nonlinear systems of differential equations and applications to reactor dynamics, Arch. Rational Mech. Anal. 5 (1960), 194211. MR 22:10285

11. P. Pucci and J. Serrin, Precise damping conditions for global asymptotic stability for nonlinear second order systems, Acta Math. 170 (1993), 275-307. MR 94i:34103

12. _ _ Precise damping conditions for global asymptotic stability for non-linear second order systems, II, J. Differential Equations 113 (1994), 505-534. CMP 95:02

13. _ Asymptotic stability for intermittently controlled nonlinear oscillators, SIAM J. Math. Anal. 25 (1994), 815-835. MR 95c:34092

14. R. A. Smith, Asymptotic stability of $x^{\prime \prime}+a(t) x^{\prime}+x=0$, Quart. J. Math. Oxford Ser. (2) 12 (1961), 123-126. MR 23:A1894

15. A. G. Surkov, On asymptotic stability of some two dimensional linear systems, Differentsial'nye Uravneniya 20 (1984), 1452-1454. (Russian) MR 85j:34098

Bolyai Institute, Aradi vértanúk tere 1, Szeged, Hungary, H-6720

E-mail address: hatvani@math.u-szeged.hu 